Introduction Bacterial vaginosis (BV) during pregnancy is linked to preterm labour and infant morbidities. Microbicides are in development for BV management, which could benefit pregnant women.

Methods Pregnant women in the US $(n=760)$ completed an online survey of attitudes about hypothetical microbicide use for BV. Conjoint analysis measured attitudes across the following dimensions: reason for microbicide use (prevention, prevention when at high risk, or treatment), product formulation (ring or gel), father of the baby's (FOB) attitudes (would want her to use the product, would not want her to use, or would not care), and ease of use (easy to insert or requires practice). Women rated 9 scenarios, each a unique combination of attributes (fractional factorial design) on a scale ranging from $0-100$ in increments of 10 . Five additional items addressed FOB involvement in use.

Results Most women were 25-35 years old (69\%), non-Hispanic White (65\%), married/living with the FOB (90\%), and had been pregnant before $(71 \%)$. Of the 702 women with complete responses, 75 gave the same rating for all scenarios (51 rating all as 80-100), precluding them from the conjoint analysis. The highest rated scenario $(M=72)$ was: for treatment, a gel, FOB wants her to use, easy to insert. The lowest rated $(M=63)$ was: for prevention, a gel, FOB doesn't want her to use, and takes practice. Conjoint analysis $(n=627)$ showed that FOB's attitudes was most important in influencing ratings (accounting for $49 \%$ of rating variability), followed by product formulation (22\%; preference for gel), reasons for use (18\%; preference for treatment), and ease of use $(11 \%)$. Most women reported the $\mathrm{FOB}$ would assist in microbicide use by being supportive (83\%), reminding them to use it $(81 \%)$, and going to the store to get it $(77 \%)$, and applying it $(58 \%)$.

Conclusions Pregnant women are accepting of microbicide use for BV; however, the opinion of the FOB will be critical for uptake. Once a microbicide is available, strategies to enhance uptake will need to foster the FOB's involvement and support.

Support: National Institutes of Health (R33AI098654)

\section{P4.103 BRAZILIAN YOUNG KEY POPULATION REACHED BY A HIV PEER-TESTING STRATEGY}

Tainah Dourado de Miranda Lobo; Clarissa Habckost Dutra de Barros; Diego Agostinho Calixto, Ana Roberta Pati Pascom. Department of STI, AIDS and Viral Hepatitis, Secretary for Health Surveillance, Ministry of Health, Brasilia - DF, Brazil

\subsection{6/sextrans-2017-053264.598}

Introduction Worldwide, young people have become increasingly vulnerable to HIV infection, especially those belonging to the key populations - the so-called "Young Key Population" (YKP). The Brazilian epidemic is largely concentrated in key populations and, over the last ten years, the aids detection rate among young males had a significant increase. Brazil, in partnership with selected NGOs, has developed an oral fluid rapid HIV peer-testing strategy tailored to key populations, called Live Better Knowing (LBK). Our aim is to provide an overview of HIV-related indexes of YKP tested on LBK.

Methods The LBK is an initiative driven in collaboration with NGOs, delivering HIV testing and prevention strategies targeted at the key populations and young people aged 15 to 24 yo, since 2013. Trained peers accessed participants in social venues for the intervention and applied a questionnaire about their sociodemographic information and risk behaviour. Results presented here are from data obtained anonymously by the strategy, between June 2015 and October 2016.

Results Among the 26010 participants aged 15 to 24yo, $65.2 \%$ were nonwhite, $65.7 \%$ reported drug use and $13 \%$ drug use and commercial sex combined. Overall, $53.8 \%$ reported condom use at last sexual intercourse and $7.9 \%$ reported STI symptoms in the last 12 months. The general HIV prevalence found was $1.2 \%$ and its distribution among cis women, transvestites, transsexual women, transsexual men, MSM and heterosexual cis men was $0.4 \%, 5.4 \%, 4.2 \%$, $0.5 \%, 3.2 \%$, and $0.5 \%$ respectively.

Conclusion YKP constitutes a high-risk population for HIV in urgent need of responses able to tackle their vulnerabilities related to the HIV acquisition. Given the combination of drug use with sex work, comprehensive harm reduction services need to be delivered. Despite the broad condom free distribution in the country, our analyses suggest the need to impact behaviours related to its use. LBK is a strategy tailored to groups who continue to face obstacles in accessing HIV services, thus, it should be expanded in order to reach out to more people.

\section{P4.104 FREQUENCY OF HIV TESTING WITH AND WITHOUT A CLINICAL CONSULTATION AMONG MEN WHO HAVE SEX WITH MEN (MSM): A RANDOMISED CONTROLLED TRIAL}

${ }^{1}$ Tim Rh Read, 'Sandra Walker, ${ }^{1}$ Eric Chow, ${ }^{1}$ Catriona Bradshaw, ${ }^{2}$ Rebecca Wigan, ${ }^{2}$ Marcus Chen, ${ }^{1}$ Christopher Fairley. ${ }^{1}$ Monash University, Melbourne, Australia; ${ }^{2}$ Melbourne Sexual Health Centre, Melbourne, Australia

\subsection{6/sextrans-2017-053264.599}

Introduction Increasing the frequency of HIV testing among populations at risk, such as MSM, enables earlier diagnosis which maximises the preventive effect of antiretroviral therapy. The requirement to attend a clinical consultation to obtain HIV testing may act as a barrier to increasing the frequency of testing and adds to the cost of additional tests.

Methods Randomised controlled trial (RCT). MSM attending Melbourne Sexual Health Centre for HIV testing were randomised $1: 1$ to an intervention allowing HIV/syphilis testing at a network of around 300 pathology centres and in the clinic, without requiring another consultation for 12 months. Controls had to attend a consultation for each HIV test as usual. During the study, both groups received four text messages recommending frequent testing. The primary outcome was the incidence of HIV testing over 12 months.

Results Of 443 men referred to the study, 13 declined participation, 8 were excluded because they were leaving Victoria and 422 were randomised. Three had positive HIV tests and were excluded from the analysis. Of 208 controls, 202 (97.1\%) and 200 of $211(94.8 \%)$ intervention group members were followed to 12 months. The intervention group had 453 tests in 205.6 person-years, incidence rate 2.2 (95\% confidence interval (CI): $2.0,2.4)$ tests per year and the control group had 432 tests during 204.0 person-years, incidence 2.1 (95\% CI: 1.9, 2.3) tests per year, incidence rate ratio (IRR) 1.04 (95\%CI: $0.89,1.2) \mathrm{p}=0.63$. The intervention group had 101 of 453 [22.3\% (95\% CI:18.5\%, 26.4\%)] tests at pathology centres without a clinical consultation.

Conclusion In this RCT, MSM who were allowed HIV/syphilis testing outside of clinical consultations did not test more 
frequently than controls who had to attend a consultation for every test. Making testing more convenient may not be sufficient to achieve increases in testing frequency. However more than one in five tests in the intervention group did not require a clinical consultation, reducing service costs.

Trial registration: ACTRN12614000760673

\section{P4.105 HIGHLY SUCCESSFUL ENGAGEMENT IN AN ACUTE HIV- INFECTION (AHI) AWARENESS CAMPAIGN AND INTERVENTION IN AMSTERDAM \& ITS YIELD OF AHI DIAGNOSES AT THE CITY'S STI CLINIC}

${ }^{1}$ Udi Davidovich, ${ }^{2}$ Maartje Dijkstra, ${ }^{3}$ Arjan Van Bijnen, ${ }^{3}$ Sjoerd Van Elsen, ${ }^{1}$ Maarten Schim Van Der Loeff, ${ }^{4}$ Fred Verdult, ${ }^{1}$ Elske Hoornenborg, ${ }^{5}$ Godelieve De Bree, ${ }^{3}$ Wim Zuilhof. ${ }^{1}$ Amsterdam Public Health Service, Amsterdam, The Netherlands; ${ }^{2}$ Academic Medical Centre, University of Amsterdam, Amsterdam, The Netherlands; ${ }^{3}$ SOA AIDS Nederland (STI Netherlands), Amsterdam, The Netherlands; "Volle Maan Communications Agency, Amsterdam, The Netherlands; ${ }^{5}$ The Amsterdam Institute for Global Health and Development (AlGHD), Amsterdam, The Netherlands

\subsection{6/sextrans-2017-053264.600}

Introduction Acute HIV infection (AHI) is associated with high HIV viral load and therefore an important factor in onward transmission. Timely detection and treatment of AHI can contribute to a better HIV prognosis and help prevent further transmission.

Methods A media campaign directed men to an online intervention (hebikhiv.nl) where visitors were trained in recognising AHI-symptoms and offered an interactive risk-assessment questionnaire using a risk score algorithm comprising behaviour and symptoms. If found at risk, men were referred to the Amsterdam STI clinic for AHI testing including a point-of-care HIV RNA test. If diagnosed with AHI, counselling and referral for immediate treatment was offered. Participation was monitored using web-statistics, and men presented for testing were questioned regarding their referral source.

Results From Aug 2015 to Dec 2016, the intervention's website was visited 150035 times by 127394 unique visitors. The online risk-assessment tool was started 96756 times and was completed in $81 \%(77,949)$ of the cases. Of those, $10 \%$ (7733) received the advice to test for AHI, and of those, 21\% (1609) downloaded the referral letter to the STI clinic. At the clinic, 209 men presented for AHI testing. Of those $57 \%$ (119) were confirmed to have arrived through the campaign. In total, 181 were eligible for testing, and in $7 \%$ (13) AHI was diagnosed. Of the 13 AHI cases, 2 were referred by the campaign, 5 by the STI clinic, 4 through own initiative, 1 by 'others', and none by a GP. All 13 were referred to start treatment within 24 hours.

Conclusion The AHI campaign succeeded in engaging a large number of MSM. The high numbers that completed the riskassessment tool points at an in-depth engagement with the intervention's material. While most men online were not found to be at risk for AHI, their engagement with the intervention could assist them to self-identify AHI in the future. The mix of referral sources among those diagnosed with AHI points at the added value of establishing a dedicated AHI testing service to attract risk cases in the community.
P4.106 INTENTION TO RE-TEST FOR STI AS A PREDICTOR FOR STI

Van Liere Gafs, Nicole Dukers-Muijrers, Christian Hoebe. Public Health Service South Limburg, Medical Microbiology Maastricht University Medical Centre, Geleen, The Netherlands

10.1136/sextrans-2017-053264.601

Introduction Guidelines advocate re-testing Chlamydia trachomatis(CT) and Neisseria gonorrhoeae(NG) positives within 312 months. Sexual risk behaviour is associated with CT/NG. Having a high intention to re-test could be a predictor for behaviour such as sexual risk behaviour. In this study we assess whether intention for re-testing before patients have received their test result is associated with CT or NG test result.

Methods Between 2014-2015, all STI clinic attendees answered the 5 point scale question "are you planning to return for testing the next year?" before CT/NG testing. Intention was categorised into very low(1), low(2), neutral(3) high(4) and very high(5). Patients were categorised in; women, heterosexual men and men who had sex with men in the past 6 months (MSM). The association between intention and CT/ NG was assessed using backward logistic regression with determinants; age, warned by (ex)partner, symptoms and number of sex partners $<6$ months. Neutral intention was the reference category.

Results Intention to re-test was lower among women and heterosexual men (median 3, inter quartile range (IQR) 2-4) compared to MSM (5, IQR 4-5) (both $\mathrm{p}<0.001)$. Overall CT prevalence was $12.9 \%(n=359)$ in women, $13.7 \%(n=216)$ in heterosexual men and $10.5 \%(n=116)$ in MSM. For NG this was $1.5 \%(n=43), 1.8 \%(n=28)$ and $12.1 \% \quad(n=134)$ respectively. In heterosexual men, having a high intention to re-test was associated with genital CT (OR1.5, 95\% CI 1.03-1.3). In women, having a very high intention was associated with genital NG (OR3.5, 95\% CI 1.3-9.0) and in MSM with anorectal NG (OR4.0, 95\% CI 1.4-11.7). Of CT positives, 48.9\% $(n=338)$ had (very) high intention, for NG positives this was $81.5 \%(\mathrm{n}=167)$.

Conclusion High intention to re-test was associated with genital CT/NG in heterosexuals and with anorectal NG in MSM. Healthcare providers could ask for and increase patients' intention to re-test during the consultation by motivational interviewing, even without the $\mathrm{CT} / \mathrm{NG}$ test result. Additionally re-testing should be promoted in diagnosed CT/NG positives, in accordance with guidelines.

\section{P4.107 HIV POST-EXPOSURE PROPHYLAXIS: PERCEPTION OF YOUNG STUDENTS FROM A UNIVERSITY OF BAHIA}

${ }^{1}$ Vinicius Nascimento dos Santos, ${ }^{2}$ Eveline Xavier, ${ }^{2}$ Maiara Timbó, ${ }^{1}$ Ana Gabriela Travassos. ${ }^{1}$ Universidade do Estado da Bahia, Salvador - BA, Brazili, ${ }^{2}$ Universidade Federal da Bahia, Salvador - BA, Brazil

\subsection{6/sextrans-2017-053264.602}

Introduction Post-Exposure Prophylaxis (PEP) is the use of antiretroviral treatment (ART) to reduce the risk of HIV infection after exposure. Interventions based on ART are recommended mostly for key populations, such as people aged 15 